\title{
Epigastric Pulsation: Prevalence and Association with Co-Morbidities in Saudi Population, a Cross- Sectional Descriptive Study
}

\author{
Manal Abdulaziz Murad ${ }^{1}$, Hoda Jehad Abousada ${ }^{2}$, Faisal Abdulaziz Almutairi ${ }^{3}$, Emad Bani Alharbi ${ }^{4}$, \\ Khalid Saad Alrashdi ${ }^{5}$, Dr. Mujib Mualla Alotaibi ${ }^{6}$, Khalil Hassan Jafari ${ }^{7}$, Mohammed Oqab \\ Althagafi $^{8}$, Majed Rabie Alhamdan ${ }^{9}$, Saud Mohammed Alotaibi ${ }^{10}$ \\ ${ }^{1}$ Assistant Professor of Family Medicine, Consultant Family Medicine, Family Medicine Department, King Abdulaziz \\ University, Jeddah, Mmurad@kau.edu.sa \\ ${ }^{2}$ Corresponding Author: KSA (Postal Address: Jeddah, 22338; dr.huda1992@outlook.com) \\ ${ }^{3}$ Student at Qassim University, KSA; faisal90511@gmail.com \\ ${ }^{4}$ Medical student at Qassim University, KSA; emadbani30@gmail.com \\ ${ }^{5}$ Medical Student at Qassim University, KSA; klooode512@hotmail.com \\ ${ }^{6}$ Medical Intern, Shaqraa University, KSA; mujib-08@hotmail.com \\ ${ }^{7}$ Medical Intern, Jazan University, KSA; khalil20901@ gmail.com \\ ${ }^{8}$ Medical intern, Taif University, KSA; oga_5@hotmail.com \\ ${ }^{9}$ Physician, East Jeddah Hospital, KSA; majed.alhamdan.3@gmail.com \\ ${ }^{10}$ Medical Intern, Shaqra University, KSA; Saudm10@outlook.com
}

Corresponding author: Hoda Jehad Abousada; Email: dr.huda1992@outlook.com

Received: 30 November 2020;

Accepted: 16 December 2020;

Published: 20 December 2020

\begin{abstract}
Epigastric pulsation is feeling of pulsation between the xiphoid process and the umbilicus. It can be found normally in population (especially in thin lean people) or abnormally as a sign or a symptom of other diseases, such as heart, liver, and aortic disease.

This descriptive study, using a questionnaire, assesses the prevalence of epigastric pulsation among 878-case sample, the association with other diseases especially DM, smoking history and obesity. Prevalence was $51.7 \%$. Most common disease to be associated with epigastric pulsation is gastrointestinal disease. No association was found with DM. Positive association was found between smoking history and BMI, and the feeling of epigastric pulsation.
\end{abstract}

Keywords: Epigastric pulsation, abdominal aortic aneurysm, diabetes, smoking, obesity.

\section{Introduction}

Epigastric pulsation is defined as feeling a pulsation in the upper abdomen, just below the xiphoid process, and above the level of the umbilicus ${ }^{[1]}$. Epigastric pulsation can be found normally in thin people, and abnormally in several pathological conditions, most importantly and commonly: Abdominal aortic aneurysm ${ }^{[2]}$. Other less common causes of epigastric pulsations include: liver vascular tumors, and right heart failure or valvular disease.

Abdominal Aortic Aneurysm (AAA) is a localized enlargement of the abdominal aorta such that the diameter is greater than $3 \mathrm{~cm}$ or more than $50 \%$ larger than normal ${ }^{[3]}$. Symptoms and signs of are either non-sensitive, non-specific, or not found. Most AAAs are found incidentally during work-up for chronic back pain, or kidney stones.

Evaluation of a patient with epigastric pulsation varies depending on the history of the patients and their risk factors. An epigastric pulsation in a thin healthy young adult is considered normal, while the same sign in a patient with known AAA is a case of emergency, and should be evaluation as ruptured AAA until proven otherwise $^{[1]}$.

\section{Literature review}

According to literature, there are no studies addressing the prevalence of epigastric pulsations per se, nor its association with other comorbidities. Literature focuses on AAA instead. 
According to a literature review conducted in 2014, AAA is a common disease in Western Countries, especially among males older than 65 -years (4-7\% prevalence), although the prevalence is decreasing in the last years ${ }^{[4]}$.

Risk factors for AAA include: age older than 65 years, male sex, and smoking history ${ }^{[5]}$. Another study suggests female sex, and high Mean arterial pressure as potential risk factors for mechanical rupture of AAA ${ }^{[11]}$. More than $90 \%$ of AAA patients have a smoking history at some point of their lives ${ }^{[6]}$. Other risk factors of AAA include: Alcohol, hypertension ${ }^{[6]}$, genetics: familial predisposition, male predominance, and enzyme deficits [7], connective tissue syndromes like Ehler's-Danlos syndrome, relapsing polychondritis and polyexanthoma elasticum ${ }^{[8]}$.

Another condition associated with AAA is Atherosclerosis, which is considered to be a risk for progression of AAA, but doesn't explain the development of AAA ${ }^{[8]}$.

As for association of AAA with other comorbidities, diabetes mellitus could be a protective factor against development of AAA according to a systemic review for English-literature articles up to September 2009. In this review, prevalence of diabetes in AAA patients ranged from 6 to $14 \%$. The prevalence of diabetes in control patients without AAA ranged from $17 \%$ to $36 \%$. Pooled analysis pointed to decrease in diabetes rates among patients with AAA, compared to those without ${ }^{[9]}$. In another Danish case-control study conducted from 1996 until 2016 on more than 3000 participants, diabetes was not found to increase risk of ruptured AAA, nor its 30-day mortality ${ }^{[10]}$. In another metanalysis, non-diabetic state was found to be protective against AAA ${ }^{[14]}$.

Smoking, however, according to a clinical research study conducted in France on more than 8000 patients, it was found that smoking is highly associated with development and growth of $\mathrm{AAA}$, and has a twofold rupture risk as well ${ }^{[12]}$. Addressing variation between the two sexes, a systematic review conducted in 2015 to follow the evidence of association between AAA and women concluded that female smokers are more likely to have AAA, and have more rupture risk ${ }^{[13]}$.

Another aspect of literature to be reviewed is that addressed to the association between the BMI and AAA development and rupture risk. According to the Society for Vascular Surgery practice guidelines, higher height of patients can be associated with higher risk for developing AAA ${ }^{[15]}$. Another review of association between obesity and AAA presence and growth, 8 studies were reviewed, and five of them were assessing the association between BMI and AAA. Three of the five studies found a positive association between increased BMI and AAA, while the other two concluded that there no relationship between increased BMI and AAA ${ }^{[16]}$. As for central obesity correlation with AAA, in a clinical research addressing that correlation, the researcher reviewed conflicting evidence regarding the relationship between are central obesity and AAA. The researcher addressed a conflict in studies assessing the correlation between AAA and central obesity. This analytical study ended up supporting lack of relationship between AAA and central obesity ${ }^{[16]}$.

\section{Aim}

To determine prevalence of Epigastric Pulsation.

\section{Objectives}

- To determine the associated disease with epigastric pulsation

- $\quad$ To determine the relationship between DM and epigastric pulsation.

- To determine the relationship between smoker and epigastric pulsation.

- To determine the relationship between BMI and epigastric pulsation.

\section{Methodology}

\section{Study design}

This is an analytical cross-sectional study.

\section{Study Setting and period}

This is an analytical cross-sectional study conducted in kingdom of Saudi Arabia (General population) from 29/7/2020 till 15/11/2020.

\section{Study population and sampling}

General population.

Study participants and sampling method

Randomised sample from general population.

Inclusion criteria; General population.

Exclusion criteria; none.

\section{Sampling size}

878

\section{Measurements}

\section{Explanatory variables}

1. Sociodemographic characteristics: age, gender and nationality.

2. Disease-related information: BMI, Smoker patient and DM patient.

\section{Results}

\section{Basic and Epidemiological Data}

As shown in table (1), sample size is 878 cases, with no missing cases in any of the results. Distribution according to age group is as follows: less than 30 years: 603 cases $(68.7 \%), 30-40$ years: 186 cases $(21.2 \%)$, above 40 years: 89 cases $(10.1 \%), 315$ cases $(40$ $\%)$ are males, while 527 cases $(60 \%)$ are females. Of 878 cases, $678(77.2 \%)$ are non-smokers, $155(17.7 \%)$ are smokers, and 45 $(5.1 \%)$ are ex-smokers. As for comorbidities, most cases $(651$ $(74.1 \%)$ ) have no comorbidities. However, 20 cases $(2.3 \%)$ have diabetes mellitus, 14 cases $(1.6 \%)$ have cardiovascular diseases, and 50 cases $(5.7 \%)$ have Respiratory disease, 79 cases $(9 \%)$ have Gastrointestinal disease, 26 cases (3\%) have nervous system disease, 29 cases $(3.3 \%)$ have high blood cholesterol, 74 cases $(8.4$ $\%)$ have other comorbidities. Among participants, 454 (51.7\%) had experienced a feeling of epigastric pulsation.

Tables 1: Characteristics of respondents

\begin{tabular}{|l|l|l|}
\hline Sample & $\mathbf{8 7 8}$ cases & $603(68.7 \%)$ \\
\hline \multirow{2}{*}{ Age groups } & Below 30 years & $186(21.2 \%)$ \\
\cline { 2 - 3 } & $30-40$ years & $89(10.1 \%)$ \\
\cline { 2 - 3 } & Above 40 years & \\
\hline
\end{tabular}




\begin{tabular}{|l|l|l|}
\hline \multirow{2}{*}{ Gender } & Male & $315(40 \%)$ \\
\cline { 2 - 3 } Smoking & Female & $527(60 \%)$ \\
\hline & Non-smoker & $678(77.2 \%)$ \\
\cline { 2 - 3 } & Smoker & $155(17.7 \%)$ \\
\hline \multirow{5}{*}{ Comorbidities } & Ex-smoker & $45(5.1 \%)$ \\
& No comorbidities & $651(74.1 \%)$ \\
\cline { 2 - 3 } & Diabetes mellitus & $20(2.3 \%)$ \\
\cline { 2 - 3 } & Cardiovascular diseases & $14(1.6 \%)$ \\
\cline { 2 - 3 } & Respiratory disease & $50(5.7 \%)$ \\
\cline { 2 - 3 } & Gastrointestinal disease & $79(9 \%)$ \\
\cline { 2 - 3 } & Nervous system disease & $26(3 \%)$ \\
\cline { 2 - 3 } & High blood cholesterol & $29(3.3 \%)$ \\
\cline { 2 - 3 } & Others & $74(8.4 \%)$ \\
\hline Epigastric pulsation feeling & Yes & $454(51.7 \%)$ \\
& No & $424(48.3 \%)$ \\
\hline
\end{tabular}

\section{Most common disease associated with epigastric pulsation} Among participants that reported feeling an epigastric pulsation, gastrointestinal disease was the most the common associated with these cases with a percentage of $9 \%$.

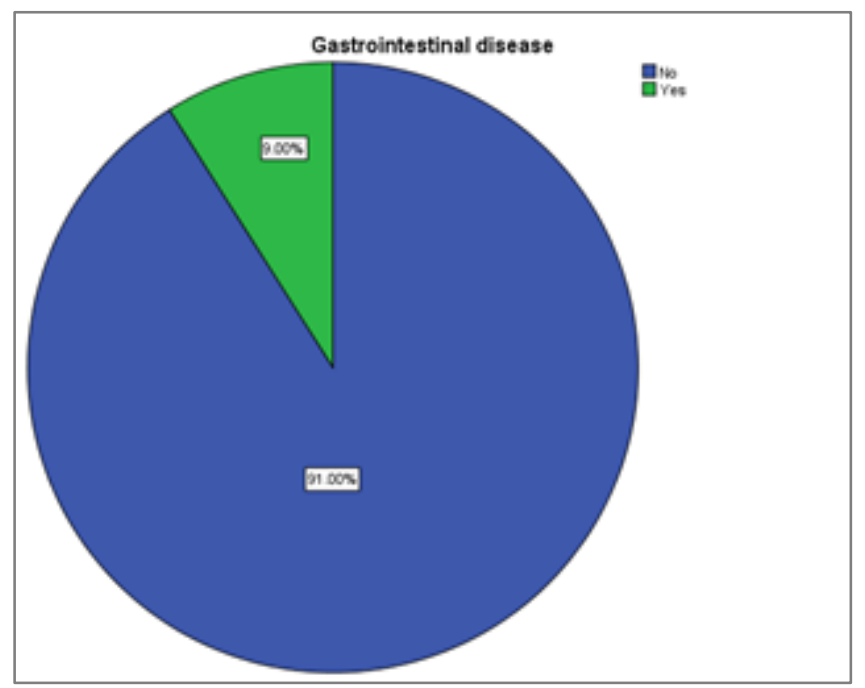

\section{Association with DM}

As shown in table 2: among respondents who reported to have epigastric pulsation, mean value of their answers reporting having DM was 0.154 (+/- .12335 SD), while mean value of their answers reporting not having DM was 0.307 (+/- .17260 SD), highlighting that DM is not associated with having a feeling of epigastric pulsation.

Table 2: Association of epigastric pulsation with Diabetes mellitus

\begin{tabular}{|l|l|}
\hline Diabetes Mellitus? & Mean (+/- SD) \\
\hline No & $0.307(+/-.17260)$ \\
\hline Yes & $0.154(+/-.12335)$ \\
\hline
\end{tabular}

\section{Association with Smoking}

However, as shown in table 3, among respondents who reported to have epigastric pulsation, mean value of their answers reporting being non-smokers was $0.5118(+/-.50023 \mathrm{SD})$, mean value of their answers reporting being smokers was 0.5355 (+/- $.50036 \mathrm{SD})$, while their the mean value of their answers reporting being exsmokers was 0.5333 (+/- .50452), highlighting that smoking is not strongly associated with having a feeling of epigastric pulsation, although this feeling occurs more frequently in smokers.
Table 3: Association of epigastric pulsation with Smoking

\begin{tabular}{|l|l|}
\hline Smoking? & Mean $(+/-$ SD) \\
\hline Non-smoker & $0.5118(+/-.50023)$ \\
\hline Smoker & $0.5355(+/-.50036)$ \\
\hline Ex-smoker & $0.5333(+/-.50452)$ \\
\hline
\end{tabular}

\section{Association with BMI}

According to our results, BMI has a higher mean value in respondents reported having an epigastric pulsation, than those who didn't.

\section{Discussion}

We present the results of a cross-sectional study based on a questionnaire distributed among a sample of Saudi population. With 878 cases, we found that prevalence of epigastric pulsation reached $51.7 \%$ of the sample. Most common disease associated with epigastric pulsation is gastrointestinal disease. DM is not associated with feeling of epigastric pulsation. Smoking is not strongly associated, yet this feeling more likely to occur in smokers. BMI was found higher in patients reporting having a feeling of epigastric pulsation.

To our knowledge, this is the first study to address the prevalence of epigastric pulsation per se. according to evidence, this feeling can occur in several entities. Normally, thin people can have epigastric pulsation feeling. Various kinds of diseases can be associated with such feeling, such as right heart disease, liver tumors, and abdominal aortic disease ${ }^{[2,3]}$.

The results of this research are highly exposed to the subjectivity of respondents, and it assesses a "symptom" that can be found in normal or abnormal conditions. That's why there are some differences between the results of this research and other literature.

In a study published in France, there was a strong association between smoking and development of AAA ${ }^{[12]}$. This supports the result of this research of addressing a higher feeling of epigastric pulsation among smokers, which could point toward an abdominal aortic disease.

With the same direction of what literature confirms, this study has not found a strong relation with DM. This goes with the literature supporting that DM has no relation with abdominal aortic disease.

A conflict of relationship with BMI is addressed in literature. In this study, respondents with higher BMI were more likely to have a feeling of epigastric pulsation, although a meta- 
analysis assessing this relationship supported the opposite, yet it has addressed that literature conflict.

\section{Conclusion}

The prevalence of epigastric pulsation equals more than half the sample. Most common disease associated with epigastric pulsation is gastrointestinal disease. DM was not found to be associated with feeling of epigastric pulsation. Smoking is not strongly associated, yet this feeling is more likely to occur in smokers. BMI was found higher in patients reporting having a feeling of epigastric pulsation.

\section{Data management and analysis}

Data were entered and analyzed using Statistical Package for the Social Sciences (SPSS) version 17. Descriptive statistics were displayed as frequencies and percentages for categorical variables. Measures of central tendencies (the median), and measures and dispersion (minimum - maximum) were used to summarize continuous variables, as the continuous variables were not normally distributed when tested by Shapiro-Wilk test. Univariate analysis was performed to investigate the association between the exposure factors with the outcome on the one hand, this was performed using Chi-squared test and Mann-Whitney test. Multivariate analysis to investigate factors independently was performed using binary logistic regression. $\mathrm{P}$ value was set at a significance level of $<0.05$.

\section{Data availability}

The data that support the findings of this study are available from the corresponding author, upon reasonable request.

\section{Conflicts of interest}

None.

\section{Funding}

Self-funded.

\section{Acknowledgments:}

To data collectors.

To participants.

\section{References}

[1] Brunicardi, F., Andersen, D., Billiar, T., Dunn, D., Hunter, J., Matthews, J., \& Pollock, R. E. (2009). Schwartz's Principles of Surgery, Ninth Edition (9th ed.). McGraw-Hill Professional. https://doi.org/10.1097/SLA.0000000000001107

[2] Moussa, O., Al Samaraee, A., Ray, R., Nice, C., \& Bhattacharya, V. (2010). A Tender Pulsatile Epigastric Mass is NOT Always an Abdominal Aortic Aneurysm: A Case Report and Review of Literature. Journal of radiology case reports, 4(10), 26-31. https://doi.org/10.3941/jrcr.v4i10.458

[3] Kent K. C. (2014). Clinical practice. Abdominal aortic aneurysms. The New England journal of medicine,
371(22),

$2101-2108$

https://doi.org/10.1056/NEJMcp1401430

[4] Svensjö, S., Björck, M., \& Wanhainen, A. (2014). Update on screening for abdominal aortic aneurysm: a topical review. European journal of vascular and endovascular surgery : the official journal of the European Society for Vascular Surgery, 48(6), 659-667. https://doi.org/10.1016/j.ejvs.2014.08.029

[5] Keisler, B., \& Carter, C. (2015). Abdominal aortic aneurysm. American family physician, 91(8), 538-543.

[6] Greenhalgh, R. M., \& Powell, J. T. (2008). Endovascular repair of abdominal aortic aneurysm. The New England journal of medicine, 358(5), 494-501. https://doi.org/10.1056/NEJMct0707524

[7] Clifton M. A. (1977). Familial abdominal aortic aneurysms. The British journal of surgery, 64(11), 765766. https://doi.org/10.1002/bjs.1800641102

[8] Rahimi, S. A. (2020, March 26). Abdominal Aortic Aneurysm: Practice Essentials, Background, Anatomy. Medscape.

https://emedicine.medscape.com/article/1979501overview\#

[9] Shantikumar, S., Ajjan, R., Porter, K. E., \& Scott, D. J. A. (2009). Diabetes and the Abdominal Aortic Aneurysm. European Journal of Vascular and Endovascular Surgery, 39(2), 200-207. https://doi.org/10.1016/j.jvs.2009.12.038

[10] Kristensen, K. L., Rasmussen, L. M., Hallas, J., \& Lindholt, J. S. (2020). Diabetes Is Not Associated with the Risk of Rupture Among Patients with Abdominal Aortic Aneurysms - Results From a Large Danish Register Based Matched Case Control Study From 1996 to 2016. European Journal of Vascular and Endovascular Surgery, 60(1), 36-42. https://doi.org/10.1016/j.ejvs.2020.02.020

[11] Lindquist Liljeqvist, M., Hultgren, R., Siika, A., Gasser, T. C., \& Roy, J. (2017). Gender, smoking, body size, and aneurysm geometry influence the biomechanical rupture risk of abdominal aortic aneurysms as estimated by finite element analysis. Journal of Vascular Surgery, 65(4), 1014-1021.e4. https://doi.org/10.1016/j.jvs.2016.10.074

[12] Lindquist Liljeqvist, M., Hultgren, R., Siika, A., Gasser, T. C., \& Roy, J. (2017b). Gender, smoking, body size, and aneurysm geometry influence the biomechanical rupture risk of abdominal aortic aneurysms as estimated by finite element analysis. Journal of Vascular Surgery, 65(4),

1014-1021.e4 https://doi.org/10.1016/j.jvs.2016.10.074

[13] Wallinder, J., Georgiou, A., Wanhainen, A., \& Björck, M. (2018). Prevalence of Synchronous and Metachronous Aneurysms in Women With Abdominal Aortic Aneurysm. European Journal of Vascular and Endovascular Surgery, 56(3), 435-440. https://doi.org/10.1016/j.ejvs.2018.05.015

[14] Bown, M. J., Sweeting, M. J., \& Brown, L. C. (2013). Surveillance Intervals for Small Abdominal Aortic Aneurysms: A Meta-analysis. Journal of Vascular Surgery, 57(6), 1720-1721. https://doi.org/10.1016/j.jvs.2013.04.019

[15] Chaikof, E. L., Brewster, D. C., Dalman, R. L., Makaroun, M. S., Illig, K. A., Sicard, G. A., Timaran, C. H., Upchurch, G. R., \& Veith, F. J. (2009). The care of patients with an abdominal aortic aneurysm: The Society 
for Vascular Surgery practice guidelines. Journal of Vascular Surgery, 50(4), S2-S49. https://doi.org/10.1016/j.jvs.2009.07.002

[16] Apoloni, R. C., Zerati, A. E., Wolosker, N., Saes, G. F., Wolosker, M., Curado, T., Puech-Leão, P., \& De Luccia,
N. (2019). Analysis of the Correlation Between Central Obesity and Abdominal Aortic Diseases. Annals of Vascular Surgery, 54, 176-184. https://doi.org/10.1016/j.avsg.2018.06.016 